Mots. Les langages du politique

\title{
La fabrique des extraterrestres
}

\section{Sébastien Poulain}

\section{CpenEdition}

\section{Journals}

Édition électronique

URL : https://journals.openedition.org/mots/19401

DOI : $10.4000 /$ mots. 19401

ISSN : 1960-6001

\section{Éditeur}

ENS Éditions

\section{Édition imprimée}

Date de publication : 1 mai 2010

Pagination : 57-66

ISBN : 978-2-84788-209-4

ISSN : 0243-6450

\section{Référence électronique}

Sébastien Poulain, «La fabrique des extraterrestres », Mots. Les langages du politique [En ligne], 92 | 2010, mis en ligne le 04 mai 2012, consulté le 22 avril 2022. URL : http://journals.openedition.org/ mots/19401; DOI : https://doi.org/10.4000/mots.19401

\section{(c) ENS Éditions}




\section{Sébastien Poulain}

\section{La fabrique des extraterrestres}

«Quand les faits manquent, les théories surabondent» (Rospars, 2007, p. 22). La distinction entre information et rumeur ne va pas de soi, surtout dans un contexte de fluidité informationnelle et d'anomie symbolique, et la multiplication des médias n'est pas le remède aux problèmes «dépressifs " (Ehrenberg, 1998) de perte de sens liés aux effets négatifs du « désenchantement du monde» (Gauchet, 1985) et de l'individualisme moderne (Durkheim, 1897). Mais au-delà de ce contexte décisif, il y a, derrière les informations non vérifiées mais remarquablement diffusées que constituent les rumeurs, des personnes qui tentent d'expliquer une réalité dont elles ont une connaissance nécessairement partielle, et qui la présentent ensuite aux autres en essayant de les convaincre de leurs théories et découvertes.

En utilisant la sociologie politique des rumeurs (Aldrin, 2005) et de la communication - de la production à la réception (Le Grignou, 2003) - et en analysant la rumeur comme un "fait social total», il s'agira ici de se demander en quoi le multimédia Radio Ici et Maintenant (lettre d'information, blog, forum, télévision) fait partie des entrepreneurs qui fabriquent des rumeurs relatives à l'apparition d'extraterrestres, et en quoi cette construction modifie le rapport ordinaire au politique et au religieux.

Si la croyance en l'existence des extraterrestres n'est envisageable qu'en termes de rumeur en l'état actuel des sciences, on pourra se demander si, d'une simple légende urbaine, elle ne tend pas à s'institutionnaliser comme religion, notamment grâce à cette radio spécialisée en spiritualité, et si cette rumeur - empreinte d'idéologie critique et libertaire, écologique et ésotérique - ne peut pas servir de répertoire d'action politique et symbolique au service des nouveaux mouvements religieux et sociaux, à l'image des religions d'avant (Déloye, 2006) et d'ailleurs (Bayart, 1993).

Carl Gustav Jung définissait la diffusion de cette croyance en les OVNI comme une «rumeur visionnaire », c'est-à-dire un «récit qui se répète et se 
raconte à travers le vaste monde, mais un récit d'une espèce particulière, se distinguant des rumeurs habituelles par le fait que son expression va jusqu'à prendre la forme de visions» (Jung, 1958, p.39). Mais sans psychologiser cette croyance, il parait évident que l'arrivée des nouvelles technologies de l'information et de la communication constitue un tournant décisif dans la manière d'écrire, répéter et raconter le récit rumoral, et a fortiori cette « rumeur visionnaire » politico-thérapeutique et scientifico-religieuse consubstantielle à la modernité. La rumeur traditionnelle - déjà en partie médiatique (Morin, 1969) - prend dès lors une tout autre ampleur accélératrice et exponentielle en raison des caractéristiques de ces nouvelles technologies - instantanéité et massivité, internationalisation et patrimonialisation, accessibilité et flexibilité - qui ont été rapidement appropriées par le personnel technophile de cette radio et des autres nouveaux entrepreneurs de rumeurs.

La propagation de cette "rumeur visionnaire», à la fois médiatique et idéologique, dépend de la fabrication et de la diffusion d'informations rumorales, aussi bien au sujet des personnes sceptiques pour les décrédibiliser que des personnes ayant vécu des contacts interplanétaires (visuels, auditifs, physiques) pour les crédibiliser. À travers l'étude, pendant cinq années, des discours et des dispositifs médiatiques liés à cette radio associative francilienne fondée en 1980, il est possible de distinguer deux types de rumeurs, a priori opposées quant à l'avenir de la société (l'une pessimiste, critique et entropique, imaginant l'autodestruction humaine, l'autre optimiste, visionnaire et utopique, imaginant une salvation extraterrestre), mais qui, en réalité, se complètent, voire s'entretiennent l'une l'autre dans leur objectif libertaire et idéaliste latent de refonder le politique en annihilant la politique : soit par son extension à une pax universalis (terriens/extra-terriens) soit par sa réduction à du technique (informatique, communicationnel, parapsychique...), et, quoi qu'il en soit, par la suppression de toute lutte de pouvoir et de toute forme de gouvernance humaine.

Ces diverses rumeurs se distinguent notamment par les personnes qu'elles visent et qui sont censées prendre en main l'avenir terrien. La rumeur pessimiste et critique s'attaque aux autorités traditionnelles fondatrices de la société moderne occidentale (l’État, l’Église, la Science), considérées comme démesurément et volontairement nocives sur les plans politique, social et religieux. La rumeur optimiste et idéaliste se focalise quant à elle sur les êtres humains qui se proclament détenteurs de pouvoirs dits paranormaux (voyants, médiums), sur les êtres extraterrestres, ainsi que sur la manière dont ces derniers pourraient sauver la planète en instaurant une ère d'harmonie pacifique, spirituelle, écologique et dépolitisée, à l'image de l'utopie New Age dont les fondateurs se sont inspirés pour la création de la radio. En effet, Didier de Plaige et Guy Skornik partent en Inde pour découvrir le bouddhisme tibétain en 1974. Didier de Plaige fonde un centre de méditation en Saône-et-Loire, traduit en 1976 
Be Here Now du psychologue psychédélique Richard Alpert, publie en 1978 Les nouveaux prophètes qui répertorie les nouveaux mouvements religieux.

Mais si la rumeur pessimiste-critique peut facilement trouver écho au sein d'une population affectée par les crises sanitaires et environnementales, militaires et humanitaires, imputées aux activités politiques et économiques, en revanche la rumeur optimiste-paranormale ne va pas de soi et doit être justifiée auprès des contemporains, dont la pensée dominante et officielle se passionne pour les récits science-fictionnels et les découvertes astrophysiques, mais stigmatise ce genre de théorie comme irrationnelle, déviante, marginale, voire sectaire. Pour combler ce déficit de légitimité, inverser les stigmates accolés au paranormal et socialiser les rumeurs et les personnes qui y croient, Didier de Plaige - dirigeant de cette radio, ancien animateur (avec Guy Skornik) d'Antenne 2 en 1977 et de France Inter en 1978 - use de différentes stratégies discursives et médiatiques. Il s'agit de construire un discours argumenté et attesté par des preuves matérielles et des témoignages, qui puisse se répandre sur le plus vaste espace public médiatique et prosélytique possible.

Pour ce qui concerne la stratégie discursive, la rumeur pessimiste doit aider à rendre crédible la rumeur optimiste. Il s'agit alors pour Didier de Plaige, qui sélectionne ses invités - donc les discours qu'ils sont susceptibles de tenir -, de jeter le discrédit sur les représentants des institutions publiques, scientifiques, médiatiques, censés disposer de l'autorité et des ressources politiques, économiques, symboliques, nécessaires pour définir l'agenda de la société contemporaine et raconter son histoire (Salmon, 2007). Ce discrédit permet de délégitimer les opinions sceptiques face au paranormal, voire d'inverser le grand partage entre vérité scientifique de la modernité civilisée et illusion magique des peuples dits «primitifs» (Lagrange, 2007). Pour cela, il est possible de dénoncer les actions et stratégies de communication (agenda, cadrage et amorçage) des autorités qui tentent de dissimuler derrière le jeu politique leurs échecs face aux enjeux sociétaux d'une réalité toujours plus imprévisible et incontrôlable (Gerstlé, 2004).

Dès lors, les distinctions entre vérité et mensonge, communication et manipulation... s'estompent. Tout peut être décrypté, relativisé, décrédibilisé, donc remis en question. Ceux qui font habituellement autorité pour dénoncer les rumeurs perdent leur légitimité et sont qualifiés par Didier de Plaige de "pseudo-scientifiques rationalistes», "primaires», "archaïques», "rétrogrades», "bornés», "manipulateurs». Le sol informationnel et cognitif devient glissant et laisse la boite de Pandore médiatique diffuser ses rumeurs. Il n'y a plus de hiérarchie entre les discours, les pratiques et les personnes (astrologues, astronomes, astronautes, ufologues...). Toutes les hypothèses sont envisageables pour donner un sens compréhensible à la réalité contemporaine dans cette sorte de désinstitutionalisation du politique. 
L'hypothèse du passage sur terre d'extraterrestres, qui peut convaincre jusqu'à $18 \%$ des enquêtés (Boy, 1993, p. 216), devient aussi crédible que n'importe quelle autre théorie. Pour justifier cette hypothèse, les ufologues s'appuient sur quatre arguments principaux:

- c'est la seule façon de sauver la Terre que les humains consument;

- les sceptiques sont manipulés par ceux qui savent (gouvernement, armée, scientifiques);

- nous ne pouvons pas être seuls dans ce vaste univers;

- il y a de nombreuses preuves matérielles et des témoignages humains, et ce depuis longtemps.

Ces quatre arguments, qu'un ufologue peut cumuler dans son répertoire de stratégie communicationnelle, aboutissent à une typologie présentant quatre postures discursives:

- un style citoyen, avec une grande conscience vis-à-vis de la société contemporaine;

- un style victimaire, avec un dégout des manipulations politiciennes;

- un style philosophique, avec des spéculations humanistes, métaphysiques et spirituelles sur l'univers;

- un style scientifique, avec une volonté objectiviste et rationaliste de décrire les découvertes ufologiques.

Si les êtres humains sont apparus sur terre, le phénomène a pu se produire différemment ailleurs et à un autre moment. Si les extraterrestres ont sur nous une supériorité physique, intellectuelle et technologique, ils peuvent, dans la terminologie française et américaine ${ }^{1}$ endogène au milieu ufologique, venir étudier notre "espèce», nous «ethnocider» ou nous sauver et passer des accords avec nos dirigeants «camouflés» par des «agences de désinformation diffamatrices» et «déboulonneuses» (debunker) qui délégitiment la "divulgation » ufologique et cachent (cover up) toute information concernant les soucoupes volantes (flying saucers), les témoignages oculaires (eyewitness testimonies), les contactés (contactees), les enlevés (abductees).

L'ufologie tend donc à être génératrice de rumeurs conspirationnistes (Pipes, 1997) qui sont des grilles d'interprétation idéologiques de la réalité rationnalisant des phénomènes sociétaux complexes (crise financière, 11 Septembre...) grâce à des explications souvent simplistes et déterministes, mais accessibles cognitivement (Festinger, 1956) et permettant d'exprimer un ressentiment antipolitique et anti-institutionnel (Enguéléguélé, 1998), voire nationaliste et raciste (Taguieff, 2005) au nom de valeurs démocratiques: transparence, liberté, vérité.

Dans un domaine où les discours et les silences sont au moins aussi

1. L'ufologie, comme science des Unidentified Flying Objects, est imprégnée de culture (médiatique) américaine, donc de son vocabulaire. Les principales observations, affaires, organisations et recherches se trouvent aux États-Unis. 
éloquents que les faits et les preuves, ce sont ces théories politiques qui nourrissent la rumeur optimiste et les espaces médiatiques de Radio Ici et Maintenant.

Pour ce qui concerne la stratégie médiatique, la radio offre à ses auditeurs des émissions de «libres antennes » où les rumeurs pessimistes sont très présentes, ainsi que des émissions où interviennent des individus prétendant posséder des pouvoirs paranormaux permettant d'aider les auditeurs et ainsi, parer aux déficits des institutions. Si ces deux types d'émission abordent rarement la problématique ufologique, elles amorcent tout de même la légitimation de cette prophétie thérapeutique religieuse extraterrestre qui dispose de trois types d'espaces médiatiques corrélés pour se déployer:

- tout d'abord, l'émission radiophonique hebdomadaire "La vague d'OVNI », unique en France par sa thématique quasi exclusive et par son professionnalisme, qui surprend l'auditeur lors de la première écoute compte tenu du sujet abordé (ton, rythme, clarté, sérieux); Didier de Plaige y interviewe des ufologues classiques, conspirationnistes ou non (chercheurs du CNES-GEPAN et du CNRS, journalistes, pilotes d'avions à la retraite), très rarement les «nouveaux ufologues» (Pinvidic, 1993, p.12) sceptiques et pragmatiques. Par exemple, l'ex-informaticien du ministère de la Défense Christel Seval, qui soutient les deux types de rumeur dans Le Plan pour sauver la Terre, est régulièrement invité pour spéculer sur les rencontres entre civilisations;

- ensuite, le blog de Didier de Plaige ${ }^{2}$ dans lequel ce dernier rend compte quotidiennement de l'abondante actualité ufologique américaine. Il y traduit des extraits d'interviews et d'articles de presse ou de sites Internet, présente les acteurs du milieu ufologique, rend compte des controverses théoriques, des résultats d'enquête, des dernières observations, annonce la diffusion de programmes audiovisuels, publie des photos d'OVNI et de leurs lieux d'atterrissage ;

- enfin, le forum de la radio3, en grande partie accaparé par l'ufologie, où les internautes débattent et s'échangent des informations audiovisuelles, commentent les blogs, à commencer par celui de Didier de Plaige, qui n'autorise pas les commentaires.

Ayant décrit le raisonnement ufologique et le réseau multimédiatique qui porte son message, le perpétue (Taïeb, 2001), voire le patrimonialise, l'institutionnalise, donc le normalise en alliant des logiques de flux et de stock informationnelles, on peut retracer la dissémination d'une rumeur à travers l'exemple de l'affaire de Stephenville, rumeur choisie ici parce qu'elle se déploie sur la

2. http://ovnis-usa.com/ (consulté le 10 décembre 2009).

3. http://icietmaintenant.fr/SMF/ (consulté le 10 décembre 2009). 
totalité de l'espace médiatique «Ici et Maintenant » et qu'elle représente bien le processus de fabrication d'une rumeur : du témoignage au complot, du local au global, du civil au militaire avec une origine étatsunienne, des stéréotypes science-fictionnels, un emballement médiatique.

Selon cette rumeur, des OVNI auraient survolé Stephenville, une ville texane proche du ranch présidentiel de Crawford4, à plusieurs reprises en janvier 2008 sous les yeux de nombreux témoins, dont un shérif. La presse locale s'empare rapidement de l'affaire, relayée par le fameux Larry King de CNN qui y consacre plusieurs émissions où sont invités des astronomes, des ufologues, des militaires, etc. Didier de Plaige tient au courant les ufologues presque quotidiennement des rebondissements de l'affaire grâce à son blog; il entre en contact avec les ufologues locaux et réalise une émission en duplex, le 5 février, avec la journaliste Linda M. Howe qui enquête sur l'affaire et en rend compte dans tous les espaces sociaux ufologiques américains : salons, médias, conférences, etc.

Les internautes du forum de la radio participent eux aussi à cette enquête collective en commentant cette actualité, en traduisant des extraits d'émissions de Larry King ou en partageant leurs propres informations, faisant de cette affaire le deuxième plus gros évènement de l'année du forum avec plus de 360 messages lus plus de 16000 fois (avant décembre 2008). Ils se demandent notamment pourquoi des informations aussi capitales ne sont pas plus relayées par les autres médias et pourquoi les autorités officielles ne prennent pas plus au sérieux l'affaire, notamment en ouvrant une enquête.

Ces ufologues-auditeurs 5 ont des profils politiques relativement hétérogènes (extrême gauche, extrême droite, écologistes, UMP, votants réguliers, abstentionnistes). Et les ufologues ont des profils sociologiques différents des auditeurs. Selon Médiamétrie, la plupart des auditeurs de l'émission font partie des catégories socioprofessionnelles inférieures (employés, ouvriers, chômeurs, chefs de petites entreprises) et ont une instruction plutôt technique. Ils sont pour les deux tiers des hommes, ont entre 35 et 49 ans ( $2 \%$ de l'audience a moins de 18 ans) et vivent plus en banlieue qu'à Paris.

D'après une enquête effectuée par Jean-Luc Lemaire, un ufologue de Charleville-Mézières, auprès de 84 ufologues parisiens réunis autour d'un

4. Les ufologues aiment accentuer ce genre d'information (tout lien avec des personnes, affaires ou lieux politiques, militaires, scientifiques) pour souligner l'importance et la vraisemblance d'un cas.

5. Ils ont été interviewés (une dizaine d'entretiens parmi les animateurs, invités et auditeurs) mais aussi observés sur une période de cinq ans : pendant les conférences des Repas ufologiques organisées le premier mardi du mois au restaurant Casino de la Défense par des personnes elles-mêmes régulièrement invitées à Radio Ici et Maintenant; pendant les discussions autour d'un verre qui suivent les Repas ufologiques où est commentée la conférence, où est discutée l'actualité politique et où les tensions ufologiques laissent place au consensus amical de la vie de tous les jours; pendant plusieurs émissions réalisées au sein de la station; pendant de nombreuses émissions réalisées en public et en direct de la librairie de l'Inconnu, rue du Cherche-Midi à Paris. 
repas, leur profil type correspond à un homme d'âge mûr mais encore en activité et qui se présente comme «documentaliste » en matière d'ufologie. Quant aux ufologues-auditeurs, ils vivent leur passion pour la politique, la science et le paranormal comme une occasion festive ou sérieuse de se socialiser, se distraire, se moquer, dénoncer, enquêter, militer... et ainsi sortir d'une marginalité souvent revendiquée, mais aussi subie.

Leurs croyances sont variées et variables et mêlent rationnel et «irrationnel», information et désinformation, politisation et dépolitisation. Ils peuvent éprouver une fascination pour le "vécu mythique ${ }^{6}$ extraterrestre (Méheust, 1985) qui a ses sources dans la culture médiatique, technologique et science-fictionnelle, voire dans les archétypes de l'«inconscient collectif»" moderne (Jung, 1958), mais doivent aussi faire face à une réalité quotidienne plus prosaïque. Et cette équivoque se retrouve dans le degré d'adhésion, c'est-à-dire le degré d'ouverture ou de clôture cognitive, à la prophétie radiophonique New Age puisque les plus passionnés et militants doivent faire leur chemin entre consonances et dissonances cognitives (Festinger, 1956) : «Il doit forcément y avoir quelque chose derrière tout ça, ce n'est pas possible.»

Mais quelle que soit l'intensité de leur foi, la plupart souhaitent apporter leur pierre à l'édification de cette religion de substitution «néo-évhémériste» (Renard, 1988, p.116-117) ou de ce «religieux sans religion» (Vieillard-Baron, 2001), tant la nébuleuse ufologique manque d'unité et de stabilité ecclésiale : autorité, lieu, doctrine, rite... En effet, l'émiettement du politico-religieux dû au désenchantement du monde laisse à la société civile un espace de spéculation illimitée et un marché du religieux ouvert pour des tentatives millénaristes de refondation de la société, à l'image de cette exobiologie qui n'a pour but que d'attester l'utopie d'une possible exopolitique. Le «merveilleux technologique » extraterrestre (Méheust, 1985) doit aider à réenchanter la manière de penser l'univers en spiritualisant, subjectivant et anthropomorphisant la matière céleste grâce à une injection rumorale de sens et d'intention, de magie et de mystère, de sacré et de spirituel, de vie et de joie. La Science - trop rationaliste, objectiviste, techniciste - n'ayant pas mieux fait que l'Église - trop hiérarchique, matérialiste, manipulatrice-pour expliquerle pourquoi des choses, le paranormal extraterrestre se nourrit de "syncrétisme scientifico-religieux» (Renard, 1988, p.116) pour répondre aux déceptions et frustrations, peurs et solitudes, incompréhensions et interrogations de la modernité, et recycler l'idéal nostalgique d'unité politico-religieuse des hommes, entre eux et avec l'univers. Dès lors, les rumeurs de «méga-complots», plus que des béquilles cognitives, sont des tentatives pour réhabiliter le politique à un niveau «supérieur » en promouvant une «grande politique » magico-scientifique dépolitisée

6. Pour Bertrand Meheust, le «vécu mythique» est l'ensemble des processus qui incarnent les mythes d'une culture dans la réalité. 
et menée par des surhommes cosmiques. Cet altermondialisme révélé devrait accoucher de citoyens universels libérés de toutes pesanteurs et salissures, contraintes et blocages qui sclérosent et étouffent la société.

Ainsi, cette interprétation revitalisante du réel innerve toute une parahistoire faite de multiples interventions stellaires (Atlantide, pyramides égyptiennes, apparitions mariales) censées attester de la plausibilité de la prophétie paramédicale. Dans ce processus étiologico-thérapeutique New Age visant le "bien-être» des humains, les macrothérapeutes extraterrestres (à l'image des dieux et esprits) fourniraient des macrosolutions spiritualo-matérielles et technico-informatiques à l'échelle universelle pour résoudre les fiascos des mésopolitiques, à l'échelle nationale, des mésothérapeutes (institutions politiques, religieuses, scientifiques) que les microthérapeutes (chamans, ufologues, numérologues) ne cessent de dénoncer sans pouvoir les juguler, en raison de leur faible nombre et de leurs micropouvoirs magiques à l'échelle individuelle. Dans cette métamorphose du politico-religieux, les prophètesufologues de cette institution médiatique «montreuse» (Neveu, 2006) de "bien-être» New Age attendent donc des messies-extraterrestres thaumaturges une action révolutionnaire et salutaire, et utilisent cette radio prophylactique (Poulain, 2008) pour collaborer à la fabrication et la diffusion d'une rumeur mythologique, cathartique et utopique, et cultiver ainsi l'espoir d'une vie meilleure dans un monde meilleur.

Cette radio remplirait alors un rôle - à la fois relatif, compte tenu de son audience et du degré d'adhésion des auditeurs, et ambigu de par l'anarchisme technologique et spirituel promu - de remise en ordre du chaos symbolique contemporain, de façon similaire à une religion dont elle partage certaines caractéristiques : des dieux extraterrestres (qui veilleraient sur les terriens à l'image des Ummites de la planète Ummo, qui auraient envoyé 6 ooo pages de courrier aux quatre coins de la planète depuis 1962 pour prévenir des dangers écologiques), un clergé ufologique (allant par exemple, au sein de la radio, du simple pasteur-animateur jusqu'au pape-directeur de la station en passant par tous les exégètes-invités), des adeptes-audiovisuels (qui font le succès des ouvrages, revues, émissions, blogs et films), des lieux ecclésiastiques de prière, de communion et de pèlerinage (médias, conférences, librairies, repas ufologiques, associations, salons), une date fondatrice (Roswell, Nouveau Mexique, 1947) avec des sources transcendantes (la foi ufologique étant souvent inexpliquée, involontaire et soudaine), syncrétiques (sélectionnées parmi des influences aussi bien religieuses et scientifiques que médiatiques et culturelles) et immémoriales ("anciens astronautes», Atlantes, Mayas, Égyptiens), des traditions, conflits et courants doctrinaux (entre les ufologues sceptiques, classiques, archéologues, conspirationnistes, orthodoxes), des saints miraculeux touchés par la grâce stellaire (témoins, contactés, enlevés). 
La rumeur fragmentée et pluridimensionnelle portant sur les extraterrestres et la manière dont elle se fabrique interrogent donc tout autant le religieux que le politique. Les citoyens, qui baignent dans un océan de politisation et dépolitisation gouverné par les lois structurelles de la sociologie (démographiques, sociales, économiques), «braconnent» une relation complexe au politique qui peut s'opérer « dans la logique et les limites des intérêts personnels immédiats» (Gaxie, 2002, p. 225), mais qui peut constituer aussi une véritable herméneutique de la vie politique ordinaire malgré le caractère parfois farfelu et peu fondé de ses idéologies et utopies. On s'aperçoit donc de ce qu'il y a d'heuristique, pour l'épistémologie politologique, dans le fait de déplacer des «perspectives vers les formes marginales, périphériques ou déviantes d'activité politique» pour «mieux faire ressortir la logique de construction et la dynamique d'évolution de l'ordre politique»(Chevallier, 1998, p.413). Ces microrécits, pour minoritaires, instables et divers qu'ils soient, n'en hantent et n'en interrogent pas moins l'imaginaire, le refoulé et le fonctionnement de la société contemporaine. Leurs grouillements nerveux et nébuleux permettent jusqu'à un certain degré de contourner ou subvertir, au moins symboliquement, les formes ordinaires de la domination sociale. Ils créent et entretiennent de façon plus ou moins formelle des schémas intellectuels, pas uniquement exutoires, de contestation, résistance ou dissidence, grâce à des répertoires d'action collective soutenus par des réseaux sociaux plus ou moins distendus, flexibles et institutionnalisés. Il n'est pas aisé de dessiner les contours conceptuels de ce qui se trouve à la croisée des nouveaux mouvements sociaux, culturels et religieux, mais leur manière, consciente ou inconsciente, d'entrer en interaction quotidiennement avec la chose publique aide à éclairer les manières de dire et de faire de la politique aujourd'hui.

\section{Références}

ALDRIN Philippe, 2005, Sociologie politique des rumeurs, Paris, PUF.

AlPERT Richard, 1976, Remember, ici et maintenant. Namasté! Paris, R. Dumas (Ram Dass, 1971, Remember, Be Here Now, San Cristobal, Lama Foudation).

BAYART Jean-François, 1993, Religion et modernité politique en Afrique noire. Dieu pour tous et chacun pour soi, Paris, Karthala.

Boy Daniel, Mıchelat Guy, 1993, «Premiers résultats de l'enquête sur les croyances aux parasciences", La pensée scientifique et les parasciences, Paris, Albin Michel.

Chevallier Jacques, 1998, "Synthèse », La politique ailleurs, E. Darras éd., Paris, PUF, CURAPP.

Deloye Yves, 2006, Les voix de Dieu. Pour une autre histoire du suffrage électoral : le clergé catholique français et le vote, xIXe-xxe siècle, Paris, Fayard.

DurkheIm Émile, 1995 [1897], Le suicide. Études de sociologie, Paris, PUF.

Ehrenberg Alain, 1998, La fatigue d'être soi. Dépression et société, Paris, Odile Jacob. 
En GuÉLÉGUÉLÉ Maurice, 1998, «La “disparition des sexes” au Cameroun », La politique ailleurs, E. Darras éd., Paris, PUF, CURAPP.

Festinger Léon, Riecken Hank, Schachter Stanley, 1993 [1956], L'échec d'une prophétie, Paris, PUF.

GAUCHET Marcel, 1985, Le désenchantement du monde. Une histoire politique de la religion, Paris, Gallimard.

GAXIE Daniel, 2002, «Les critiques profanes de la politique», Juger la politique. Entreprises et entrepreneurs critiques de la politique, J.-L. Briquet, P.Garraud éd., Rennes, Presses universitaires de Rennes.

GerstLÉ Jacques, 2004, La communication politique, Paris, Dalloz / Armand Colin.

JUNG Carl Gustav, 1958, Un mythe moderne, Paris, Gallimard.

LAG RAN Ge Pierre, 2007, Ovnis. Ce qu'ils ne veulent pas que vous sachiez, Paris, Presses du Châtelet.

Leduc Jean-Marie, PlaIGE Didier (DE), 1978, Les nouveaux prophètes, Paris, Buchet/ Chastel.

Le Grignou Brigitte, 2003, Du côté du public. Réceptions et usages de la télévision, Paris, Economica.

MÉHEUST Bertrand, 1985, Soucoupes volantes et folklore, Paris, Mercure de France.

Morin Edgar, 1969, La rumeur d'Orléans, Paris, Le Seuil.

NeVEu Érik, 2006, Une société de communication?, Paris, Montchrestien.

PINVIDIC Thierry, 1993, "Introduction», OVNI. Vers une anthropologie d'un mythe contemporain, t. Pinvidic éd., Paris, Heimdal.

PIPES Daniel, 1997, Conspiracy. How the paranoid style flourishes and where it comes from, New York, Free Press.

Poulain Sébastien, 2008, "Guérir de la société grâce à la radio. Usages des libres antennes de Radio Ici et Maintenant», Istanbul, AISLF [en ligne] [http://w3.aislf. univ-tlse2.fr/gtsc/DOCS_SOCIO/istambul/Actes_AISLF_GT13_Istambul_2008. pdf], consulté le 2 décembre 2009.

RENARD Jean-Bruno, 1988, Les Extraterrestres. Une nouvelle croyance religieuse?, Paris, Le Cerf.

Rospars Jean-Pierre, 2007, "Un échec de la science», Inforespace, ${ }^{\circ}$ 114-115.

SALMON Christian, 2007, Storytelling. La machine à fabriquer des histoires et à formater les esprits, Paris, La Découverte.

SEVAL Christel, 2005, Le Plan pour sauver la Terre, Agnières, JMG.

TAgUIEFF Pierre-André, 2005, La foire aux illuminés. Ésotérisme, théorie du complot, extrémisme, Paris, Mille et une Nuits.

TAḯb Emmanuel, 2001, «Persistance de la rumeur. Sociologie des rumeurs électroniques », Réseaux, $\mathrm{n}^{\circ} 106$.

VIEILLARD-BARon Jean-Louis, 2001, La religion et la cité, Paris, PUF. 